\title{
Healthy diet in child obesity
}

\section{Proceeding}

Childhood obesity is one of the most serious public health challenges of the 21 st century. The problem is global and is steadily affecting many low- and middle-income countries, particularly in urban settings. The prevalence has increased at an alarming rate. Globally, in 2016 the number of overweight children under the age of five is estimated to be over 41 million. Today, nearly 1 out of 4 children and teens in developed countries are overweight or obese. Those extra pounds put kids at risk for developing serious health problems, including diabetes, heart disease, and asthma. Childhood obesity also takes an emotional toll. Overweight children often have trouble keeping up with other kids and joining in sports and activities. Other kids may tease and exclude them, leading to low self-esteem, negative body image, and even depression. These issues can be solved with proper education and support, children can learn healthier ways to cope with their problems, prepare meals, and stay active. This support must come from the adults in their lives: parents, teachers, and other caregivers. Help your children stay healthier for longer by preparing nutritious foods for them and encouraging them to get plenty of exercise.

Childhood obesity is one of the most serious public health challenges of the 21 st century. The problem is global and is steadily affecting many low- and middle-income countries, particularly in urban settings. The prevalence has increased at an alarming rate. Globally, in 2016 the number of overweight children under the age of five is estimated to be over 41 million. Almost half of all overweight children under 5 lived in Asia and one quarter lived in Africa.

Today, nearly 1 out of 4 children and teens in developed countries are overweight or obese. Those extra pounds put kids at risk for developing serious health problems, including diabetes, heart disease, and asthma. Childhood obesity also takes an emotional toll. Overweight children often have trouble keeping up with other kids and joining in sports and activities. Other kids may tease and exclude them, leading to low self-esteem, negative body image, and even depression.

But there's plenty you can do to help your kids. Diagnosing weight problems and obesity in children as early as possible can reduce their risk of developing serious medical conditions as they get older. And by getting the whole family involved, you can break the cycle of weight problems and obesity, boost your children's physical and mental health, and help them establish a healthy relationship with food that will last a lifetime. Whatever your children's weight, let them know that you love them and that all you want to do is help them be healthy and happy.

Children grow at different rates at different times, so it is not always easy to tell if a child is overweight. Body mass index (BMI) uses height and weight measurements to estimate how much body fat a child has. However, while BMI is usually a good indicator, it is NOT a perfect measure of body fat and can even be misleading at times when children are experiencing periods of rapid growth. If your child registers a high BMI-for-age measurement, your health care provider may need to perform further assessments and screenings to determine if excess fat is a problem.
Volume 8 Issue 2 - 2018

Azra Roohi
Consultant Dietitian, Nutritionist and trainer,AI Kafeel Super
Speciality Hospital, Iraq

Correspondence: Azra Roohi, Consultant Dietitian, Nutritionist and trainer, Al Kafeel Super Speciality Hospital, Iraq, Tel 00964783 I805506, Email azra.roohi@gmail.com

Received: March 06, 2018| Published: April 05, 2018

Understanding how children become overweight in the first place is an important step toward breaking the cycle. Most cases of childhood obesity are caused by eating too much and exercising too little. Children need enough food to support healthy growth and development. But when they take in more calories than they burn throughout the day, the result is weight gain. Causes of weight problems in children may include:

i. Busy families cooking at homeless and eating out more.

ii. Easy access to cheap, high-calorie fast food and junk food.

iii. Bigger food portions, both in restaurants and at home.

iv. Kids consuming huge amounts of sugar in sweetened drinks and hidden in an array of foods.

v. Kids spending less time actively playing outside, and more time watching TV, playing video games, and sitting at the computer.

vi. Many schools eliminating or cutting back their physical education programs.

Healthy habits start at home. The best way to fight or prevent childhood obesity and weight problems is to get the whole family on a healthier track. Making better food choices and becoming more active will benefit everyone, regardless of weight.

\section{Make healthier food choices}

While you may need to make major changes to your family's eating habits, changing everything at once usually leads to cheating or giving up. Instead, start by making small, gradual steps-like adding a salad to dinner every night or swapping out French fries for steamed vegetables-rather than one big drastic switch. As small changes become habit, you can continue to add more healthy choices.

a. Eat the rainbow: Serve and encourage consumption of a wide variety of fruits and vegetables. This should include red (beets, tomatoes), orange (carrots, squash), yellow (potatoes, bananas), green (lettuce, broccoli) and so on-just like eating a rainbow.

b. Make breakfast a priority: Children who eat breakfast are less likely to be overweight or obese than those who skip the first meal of the day. It's important to focus on healthy choices, though, like oatmeal, fresh fruit, whole grain cereal high in fiber and low in sugar, and low-fat milk instead of sugary cereals, donuts, or toaster pastries. 
c. Look for hidden sugar: Reducing the amount of candy and desserts you and your child eat is only part of the battle.

d. Sugar is also hidden in foods as diverse as bread, canned soups, pasta sauce, instant mashed potatoes, frozen dinners, low-fat meals, fast food, and ketchup. The body gets all it needs from sugar naturally occurring in food-so anything added amounts to nothing but a lot of empty calories. Check labels and opt for low sugar products and use fresh or frozen ingredients instead of canned goods.

e. Schedule regular meal times: The majority of children like routine. If your kids know they will only get food at certain times, they will be more likely to eat what they get when they get it.

f. Limit dining out. If you must eat out, try to avoid fast food.

g. Don't go no fat, go good fat: Not all fats contribute to weight gain. So instead of trying to cut out fat from your child's diet, focus on replacing unhealthy fats with healthy fats.

h. Avoid trans fats: that are dangerous to your child's health. Try to eliminate or cut back on commercially-baked goods packaged snack foods, fried foods, and anything with "partially hydrogenated" oil in the ingredients, even if it claims to be trans fat-free.

i. Add more healthy fats: that can help a child control blood sugar and avoid diabetes. Unsaturated or "good" fats include avocados, olive oil, nuts, fatty fish, flaxseed and spinach.

j. Choose saturated fat wisely: The USDA recommends limiting saturated fat to 10 percent of your child's daily calories. Focus on the source of saturated fats consumed: A glass of whole milk or natural cheese rather than a hot dog, donut, or pastry, for example, or grilled chicken or fish instead of fried chicken.

k. Be smart about snacks and sweet food: our home is where your child most likely eats the majority of meals and snacks, so it is vital that your kitchen is stocked with healthy choices.

1. Look for hidden sugar: Reducing the amount of candy and desserts you and your child eat is only part of the battle. Sugar is also hidden in foods as diverse as bread, canned soups, pasta sauce, instant mashed potatoes, frozen dinners, and low-fat meals. The body gets all it needs from sugar naturally occurring in foodso anything added amounts to nothing but a lot of empty calories. Check labels and opt for low sugar products and use fresh or frozen ingredients instead of canned goods.
m.Don't ban sweets entirely: While many kids' consume too much sugar, having a no sweets rule is an invitation for cravings and overindulging when given the chance. Instead, limit the amount of cookies, candies, and baked goods your child eats and introduce fruit-based snacks and desserts instead.

n. Limit juice, soda, and coffee drinks: Soft drinks are loaded with sugar and shakes and coffee drinks can be just as bad. Many juices aren't any better nutritionally, so offer your child sparkling water with a twist of lime, fresh mint, or a splash of fruit juice instead.

o. Keep snacks small: Don't turn snacks into a meal. Limit them to 100 to 150 calories. Go for reduced-sugar options. When buying foods such as syrups, jellies, and sauces, opt for products labeled "reduced sugar" or "no added sugar."

p. Focus on fruit: Keep a bowl of fruit out for your children to snack on.

q. Experiment with herbs and spices: Use sweet-tasting herbs and spices such as mint, cinnamon, allspice, or nutmeg to add sweetness to food without the empty calories.

r. Check the sugar content of your kid's cereal: There's a huge disparity in the amount of added sugar between different brands of cereal. Some cereals are more than $50 \%$ sugar by weight. Try mixing a low sugar, high-fiber cereal with your child's favorite sweetened cereal, or add fresh or dried fruit to oatmeal for a natural sweet taste.Childhood obesity is a serious issue However, with proper education and support, children can learn healthier ways to cope with their problems, prepare meals, and stay active. This support must come from the adults in their lives: parents, teachers, and other caregivers. Help your children stay healthier for longer by preparing nutritious foods for them and encouraging them to get plenty of exercise.

\section{Acknowledgment}

None.

\section{Conflicts of interest}

None. 